\title{
A Novel Non-contact Self-Injection-Locked Radar for Vital Sign Sensing and Body Movement Monitoring in COVID-19 Isolation Ward
}

\author{
Cheng-Yu Tsai ${ }^{1,2} \cdot$ Nai-Chien Chang ${ }^{3} \cdot$ Hsiu-Chen Fang ${ }^{3} \cdot$ Ying-Che Chen $^{4} \cdot$ Su-Shin Lee ${ }^{5,6,7}$ (D)
}

Received: 15 July 2020 / Accepted: 5 August 2020 / Published online: 26 August 2020

(C) Springer Science+Business Media, LLC, part of Springer Nature 2020

\begin{abstract}
Background The outbreak of Coronavirus disease (COVID-19) pandemic has become the most serious global health issue. Isolation policy in hospitals is one of the most crucial protocols to prevent nosocomial infection of COVID-19. It is important to monitor and assess the physical conditions of the patients in isolation.

Methods Our institution has installed the novel non-contact wireless sensor for vital sign sensing and body movement monitoring for patients in COVID-19 isolation ward.

Results We have collected and compared data between the radar record with the nurse's handover record of two patients, one recorded for 13 days and the other recorded for 5 days. The $P$ value by Fisher's exact test were 0.139 (temperature, $P>0.05$ ) and 0.292 (heart beat rate, $\mathrm{P}>0.05$ ) respectively.

Conclusions This is the first report about the application experience of this equipment. Therefore we attempted to share the experience and try to apply this equipment in COVID-19 patients in future to offer the more reliable and safe policy.
\end{abstract}

Keywords Coronavirus disease $\cdot$ Self-injection-locked radar $\cdot$ Vital sign sensing

\section{Background}

As Coronavirus disease 2019 (COVID-19) spreads across the world, the COVID-19 pandemic has become the most serious

This article is part of the Topical Collection on Mobile \& Wireless Health.

Electronic supplementary material The online version of this article (https://doi.org/10.1007/s10916-020-01637-z) contains supplementary material, which is available to authorized users.

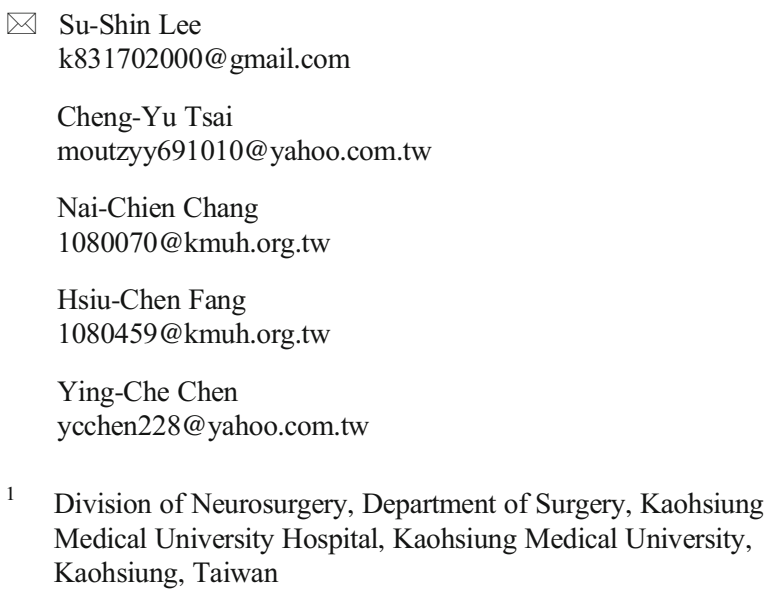

1 Division of Neurosurgery, Department of Surgery, Kaohsiung Medical University Hospital, Kaohsiung Medical University, Kaohsiung, Taiwan

global health issue and caused huge impacts on healthcare systems. Since COVID-19 is extremely transmissible, the clinical features are non-specific and cannot be easily distinguished from other causes of severe community-acquired
2 Program in Environmental and Occupational Medicine, College of Medicine, Kaohsiung Medical University and National Health Research Institutes, Kaohsiung, Taiwan

3 International medical service center, Kaohsiung Medical University, Kaohsiung, Taiwan

4 Department of Surgery, Kaohsiung Municipal Siaogang Hospital, Kaohsiung, Taiwan

5 Division of Plastic Surgery, Department of Surgery, Kaohsiung Medical University Hospital, Kaohsiung, Taiwan

6 Regenerative medicine and cell therapy research center, Kaohsiung Medical University, Kaohsiung, Taiwan

7 Department of Surgery, Faculty of Medicine, College of Medicine, Kaohsiung Medical University, Kaohsiung, Taiwan 
pneumonia. [1] The healthcare industry is suffering from the surge of COVID-19, and carrying the risks of being overwhelmed by the pandemic. For example, there is a potential risk of nosocomial transmission due to the viable virus remained on personal protective equipment (PPE) used by the healthcare staff. Zamora et al. has reported a substantial risk of self-contamination when doffing PPE. [2] In addition, since there is a higher risk of being infected or in quarantine caused by the exposure to COVID-19 from improper protections, hospital staff's morale is highly affected. Therefore, we shall utilize the new technology to protect the healthcare staff as well as maintaining proper health care service quality to the publics.

In this report, we introduce a non-contact self-injectionlocked radar (SIL Radar Technology INC., Kaohsiung, Taiwan). The SIL Oscillator's output signal is received and demodulated by a remote frequency discriminator to obtain the pulse rate information. [3] It can provide the information for vital signs and large body movements of the patient. This instrument has been used on COVID-19 patients as an auxiliary monitor system in the isolation ward.

\section{Methods}

The non-contact wireless sensor has been fixed on the ceiling of the ward. It detects and records the vital signs of the patient every five seconds automatically without physical contacts between the patient and healthcare staff. There have been several engineering research articles of the device presented by the inventors. The technologies of self-injection-locked radar and infrared light are used in non-contact detection of vital signs, included body temperature, heart rate, respiratory rate and body positioning [3-7]. As more information is collected, the software of this device is able to conduct an autocalibration to extract the displacement information, which enhances the measurement accuracy of body temperature, heart rate and respiratory rate. [5]

\section{Results}

We have collected and compared data between the radar record with the nurse's handover record of two patients, one recorded for 13 days and the other recorded for 5 days. The $P$ value by Fisher's exact test were 0.139 (temperature, $P>0.05$ ) and 0.292 (heart beat rate, $\mathrm{P}>0.05$ ) respectively.

In addition, the device can detect the human face and body movement to identify actual positions of the patient, such as lying on the bed or falling down on the floor in the ward. [8] The device has performed aforementioned function via remote wireless controlling system automatically, and $24 \mathrm{~h}$ a day uninterruptedly to ensure the comfort and privacy of the patient.

\section{Discussions}

During the COVID-19 pandemic, the emotional stress of the healthcare staff in the isolation ward is enormous. Contactless assessment of the important physiological parameters can reduce the risk of transmission of COVID-19 virus, and also boost the morale of the team members.

The camera based vital sign sensors have been developed for the past 20 years. The majority of these contactless devices are infrared thermography with a Red-Green-Blue (RGB) digital camera. The applications of these sensors are monitoring of neonates in neonatal intensive care units, monitoring of heart rate during regional anesthesia, during dialysis and in the post-anesthesia care units. [9] Since the RGB camera records the facial features of the patient, there may be violations of privacy. Due to the same issue, the device can't be installed in the toilet or shower room. On the other hand, the selfinjection-locked radar uses the wideband oscillators and extracts accurate vital signs from the received Doppler-shifted signal. There is no high-resolution image captured, and the privacy issue is minimized. (Fig. 1).

In our hospital, we have setup a self-injection-locked radar on the ceiling of the ward and its bathroom to monitor patient's vital signs. (Fig. 2) The device has been used to collect the heart rate and body temperature of the isolated patient from COVID-19. The data is consistent with the nursing handover record. The $P$ value by Fisher's exact test are temperature, $P>0.05$ and heart rate, $\mathrm{P}>0.05$ respectively. There is no statistics difference between these two sets of data. For patients in the isolation ward, the healthcare staff has to care them under PPE. However, in the report of Wang et al., the virus nucleic acid can be tested in multiple surface areas in the patient's room. [10] Therefore, utilizing the contactless radar technology allows the healthcare staff to check the heart rate and temperature of the patient from the nursing station (a clean zone) anytime. The nursing staffs no longer need to rush into the rooms to check the patients' vital signs if patients fall asleep. The continuously heart rate data can help medical staffs to rule out bradycardia situation of the patient. This can decrease the infection and transmission risk for the healthcare staff.

Furthermore, the device can detect the actual position of isolated patients via sensing movements of the human face and body. Since cough and shortness of breath are very important symptoms of COVID-19, we also could use the selfinjection-locked radar to detect the real-time movement of cough and any respiratory movement. This function can save time for the healthcare staff since there is no need to physically check on patients frequently. Another benefit is the reduced 
Fig. 1 The thermography without high resolution image of patient can protect the privacy of the patient. The heart rate and respiratory rate are listed on the right side

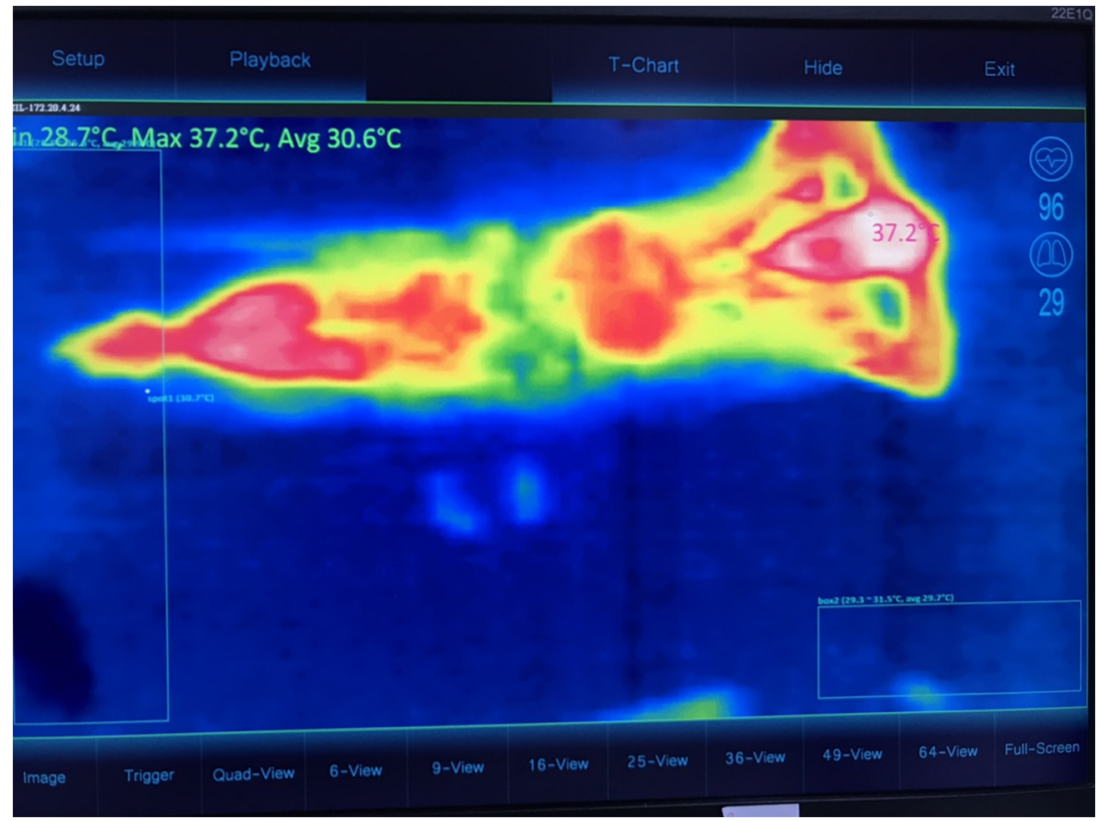

waste of PPE. The wireless feature will also provide another advantage for long period of isolation; the patient won't be "cabled" with the EKG wires. Patients under long-term isolation normally suffer from uncomfortable mental stress. The wireless device can provide comfort and privacy for them.

The device is programmed with Artificial Intelligence (AI) self-learning function. This function can detect the abnormal physical data trend, such as the pause or deceleration of respiratory rate. As aforementioned advantages and features, we

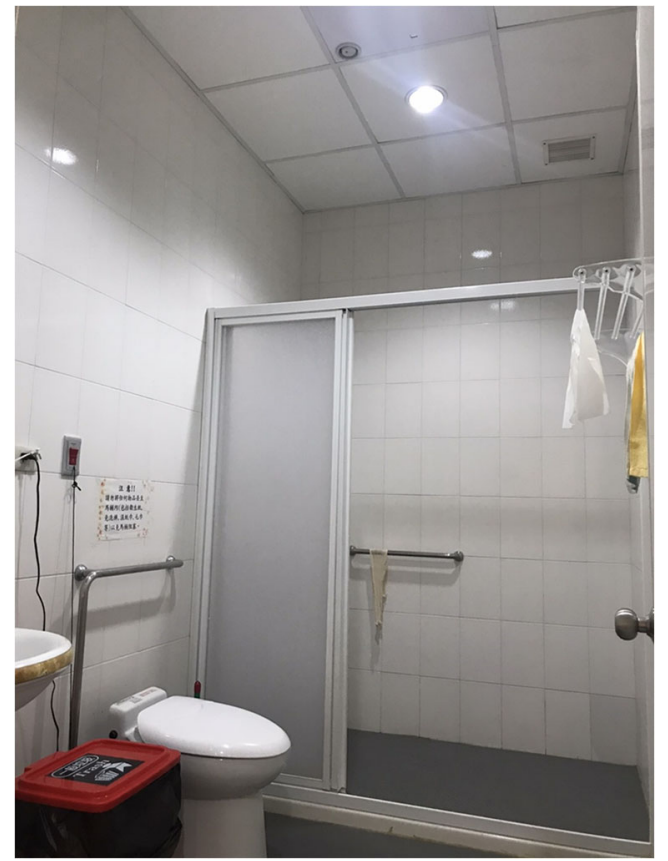

Fig. 2 One self-injection-locked radar locates on the ceiling of the water closet to monitor patient vital sign in this private area are here to share the experiences and extend the usage of this device for COVID-19 patients under isolation.

\section{Conclusions}

This is the first report about the application experience of this equipment. Therefore we attempted to share the experience and try to apply this equipment in COVID-19 patients in future to offer the more reliable and safe policy.

Acknowledgements The authors thank Peggy Tsai for English editing.

Availability of data and materials Not applicable

Authors' contributions CYT and SSL are major contributor in writing the manuscript. HCF, NCC and YCC analyzed and interpreted the patient data. All authors read and approved the final manuscript.

\section{Compliance with ethical standards}

Ethics approval and consent to participate: not applicable.

Consent for publication not applicable.

Competing interests The authors declare that they have no competing interests.

\section{References}

1. Phua J, Weng L, Ling L, Egi M, Lim CM, Vasishtha Divatia J et al. Intensive Care Management of Coronavirus Disease 2019 (COVID-19): Challenges and Recommendations. Lancet Respir 
Med. 2020 May;8(5):506-517. https://doi.org/10.1016/S22132600(20)30161-2. Epub 2020 Apr 6

2. Zamora JE, Murdoch J, Simchison B, Day AG. Contamination: a comparison of 2 personal protective systems. CMAJ 2006; 175: 249-54. https://doi.org/10.1503/cmaj.060094

3. Wang FK, Tang MC, Su SC, Horng TS. Wrist Pulse Rate Monitor Using Self-Injection-Locked Radar Technology. Biosensors (Basel). 2016 Oct 26;6(4):54-66

4. Peng KC, Lee CH, Wong DG, Wang FK, Horng TS. An injectionand Frequency-Locked Loop for Reducing phase noise of wideband oscillators. IEEE Transactions on Microwave Theory and Techniques. 2018 Mar;66(3):1374-83. https://doi.org/10.1109/ TMTT.2017.2779132

5. Tang MC, Wang FK, Horng TS. Single Self-Injection-Locked Radar With Two Antennas for Monitoring Vital Signs With Large Body Movement Cancellation. IEEE Transactions on Microwave Theory and Techniques. 2017 Dec;65(12):5324-33. https://doi.org/10.1109/TMTT.2017.2768363

6. Wang FK, Juan PH, Su SC, Tang MC, Horng TS. Monitoring Displacement by a Quadrature Self-Injection-Locked Radar With Measurement- and Differential-Based Offset Calibration Methods. IEEE Sensors Journal. 2019 Mar;19(5):1905-16. https://doi.org/10. 1109/JSEN.2018.2884729
7. Hsu CY, Chuang CY, Wang FK, Horng TS, Hwang LT. Detection of vital signs for multiple subjects by using self-injection-locked radar and mutually injection-locked beam scanning array. 2017 IEEE MTT-S International Microwave Symposium (IMS). 2017 Honololu, HI :991-4. https://doi.org/10.1109/MWSYM.2017. 8058756

8. Wang FK, Su SC, Tang MC, Horng TS. Displacement monitoring system based on a quadrature self-injection-locked radar technology. 2017 IEEE MTT-S International Microwave Symposium (IMS). 2017 Honololu, HI:1363-6. https://doi.org/10.1109/ MWSYM.2017.8058867

9. Antink CH, Lyra S, Paul M, Yu X, Leonhardt S. A Broader Look: Camera-Based Vital Sign. Yearb Med Inform 2019:102-14

10. Wang H, Mob P, Lic G, Chend P, Liua J, Wang $H$ et al. Environmental virus surveillance in the isolation ward of COVID-19. J Hosp Infect. 2020 Apr 15;S0195-6701(20)30195X. https://doi.org/10.1016/j.jhin.2020.04.020

Publisher's Note Springer Nature remains neutral with regard to jurisdictional claims in published maps and institutional affiliations. 